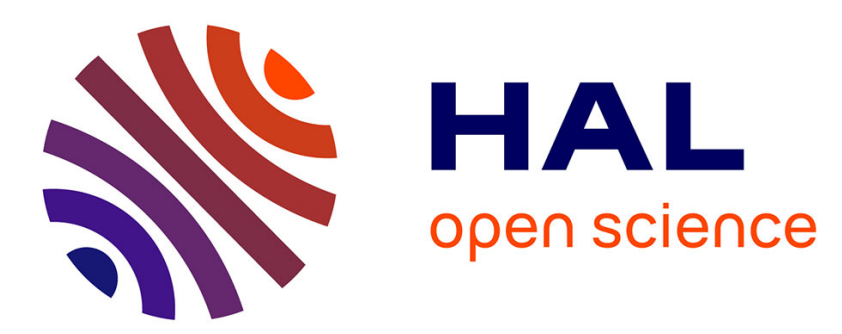

\title{
Regulation of pendulum length as a control mechanism in performing the backward giant circle in gymnastics
}

\author{
Guillaume Rao, Violaine Sevrez, Eric Berton, Reinoud J. Bootsma
}

\section{To cite this version:}

Guillaume Rao, Violaine Sevrez, Eric Berton, Reinoud J. Bootsma. Regulation of pendulum length as a control mechanism in performing the backward giant circle in gymnastics. Human Movement Science, 2009, 28 (2), pp.250-262. 10.1016/j.humov.2008.10.002 . hal-01635489

\section{HAL Id: hal-01635489 \\ https://hal-amu.archives-ouvertes.fr/hal-01635489}

Submitted on 15 Nov 2017

HAL is a multi-disciplinary open access archive for the deposit and dissemination of scientific research documents, whether they are published or not. The documents may come from teaching and research institutions in France or abroad, or from public or private research centers.
L'archive ouverte pluridisciplinaire HAL, est destinée au dépôt et à la diffusion de documents scientifiques de niveau recherche, publiés ou non, émanant des établissements d'enseignement et de recherche français ou étrangers, des laboratoires publics ou privés. 


\title{
Regulation of pendulum length as a control mechanism in performing the backward giant circle in gymnastics
}

\author{
Violaine Sevrez, Eric Berton, Guillaume Rao, Reinoud J. Bootsma* \\ UMR 6233 ISM Marey, Université de la Méditerranée, 163, avenue de Luminy, 13288 Marseille Cedex 9, France
}

\section{A R T I C L E I N F O}

\section{Article history:}

Available online 3 February 2009

PsycINFO Classification:

2330

Keywords:

Motor control

Model

Perception

\begin{abstract}
A B S T R A C T
Seven female elite gymnasts performed backward giant circles on the high bar under different conditions of loading. The magnitude ( 2 or $4 \mathrm{~kg}$ ) and location (shoulders, waist, and ankles) of load systematically influenced the overall swing duration as well as the relative timing of movements at the joints. An analysis of the mechanical constraints operating suggested that the gymnast should be considered as a pendulum of variable length. Increasing and decreasing pendulum length at appropriate phases of the swing effectively allows energy to be injected into the system, thereby compensating the energy lost to friction. A sharp negative peak in the relative rate of change of pendulum length, characteristic of the upward swing phase of all gymnasts, was found to invariably occur at a particular value of the first-order time-to-closure of the body orientation gap with respect to the vertical. The presence of this invariant suggested that the gymnasts organize their behavior on the basis of such a first-order temporal relation. (c) 2008 Elsevier B.V. All rights reserved.
\end{abstract}

\section{Introduction}

Ever since Borelli introduced mechanics into the study of living systems (Turvey, 1994), research in the domain of (human) movement has benefited much from the in-depth investigation of the anatomical, physiological, and biomechanical functioning of the different parts of the musculoskeletal system. However, due to the invasive presence of redundant degrees of freedom, movements can be performed in many different ways and mechanical principles alone cannot explain the reasons underlying the adoption of one particular pattern of movement rather than another. The processes by which the multitude of degrees of freedom are coordinated in mastering a perceptuo-motor skill (Bernstein,

\footnotetext{
* Corresponding author. Tel.: +33 4911704 36; fax: +33 491172252.

E-mail address: reinoud.bootsma@univmed.fr (R.J. Bootsma).
} 
1967) requires a control-centered rather than a mechanics-centered approach. Separately, mechanicscentered and control-centered analyses provide important yet restricted knowledge of human motion. Combining these approaches allows for a better understanding of the perceptuo-motor control strategies that are used in the regulation of the mechanically-constrained movements that constitute the physical basis of all perceptuo-motor skills. In the present contribution we examined a task that is not only highly constrained from a mechanical point of view but that also requires a particular spatiotemporal organization of the entire body: the backward giant circle on the high bar in gymnastics.

The giant circle is a gymnastic element in which the gymnast departs from a handstand position above the bar and fully rotates around it, without releasing the bar at any time. During a gymnastic exercise, giant circles are often performed in preparation of upcoming flight elements, including the final dismount. Such accelerating giant circles have received quite some attention from a performance-oriented biomechanical perspective (Arampatzis \& Brüggemann, 1999, 2001; Hiley \& Yeadon, 2003a, 2003b, 2005; Yeadon \& Hiley, 2000). However, because we seek to understand the organizational principles - rather than the biomechanical limits - underlying the execution of backward giant circles, in the present contribution we studied smooth circling movements where the goal is to return to the handstand position above the bar with minimal rotational speed.

Let us begin our analysis by considering the giant circle from a physical point of view in order to identify the mechanical constraints operating. Starting by considering the gymnast as a point mass (located at the body center of mass, BCM) rigidly attached to an axis of rotation simplifies the identification of the pertinent mechanical characteristics. In the absence of damping, the dynamics of such a simple pendulum is governed by the gravitational torque operating ( $m g r \sin \theta$, where $m$ is the gymnast's body mass, $g$ is the acceleration due to gravity, $r$ is the pendulum length, and $\theta$ is the angular deviation from the vertical). This torque results in a rotational acceleration $(\ddot{\theta})$ modulated by the moment of inertia $\left(I \ddot{\theta}=m r^{2} \ddot{\theta}\right)$. Hence, such a system is described by the following equation of motion:

$$
\ddot{\theta}+\frac{g}{r} \sin \theta=0 .
$$

When released from a given initial position $\theta_{\mathrm{i}}$ the pendulum accelerates until the mass is directly beneath the axis of rotation. At this point, the potential energy associated with the initial position has been fully transformed into kinetic energy. This kinetic energy sustains movement until the mass has risen to attain $-\theta_{\mathrm{i}}$. At this point, all kinetic energy has been transformed to potential energy. This motion would continue perpetually if no friction forces where operating, that is if mechanical energy the sum of potential and kinetic energies - were constant. In reality, all physical systems dissipate energy, inexorably causing the pendulum to stop. For our gymnast, friction is exerted by the rotation of the hands around the bar and by the resistance of air to movement of the body. Thus, when maintaining a given body configuration throughout the movement energy is lost and the gymnast will not return to the desired handstand position.

In order to fulfil the task requirements, energy thus needs to be inserted into the system. Changes in body configuration lead to changes in pendulum length. As demonstrated by Tea and Falk (1968), such changes in pendulum length $(r)$ give rise to changes in mechanical energy content. Decreasing $r$ at the lowest point of the arc through which the BCM moves and increasing $r$ at the highest point(s) effectively allows energy to be pumped into the system. Applying this logic to the giant circle in gymnastics, Bauer (1983) suggested that trajectories with two sectors of piecewise constant $r$ (long from handstand to the lowest point and short from there back to the handstand position) would constitute an optimal strategy. Yet, empirical studies have demonstrated that the movement patterns of gymnasts performing giant circles do not reveal a rapid flexion at the lowest point followed by a rapid extension at the highest point (Arampatzis \& Brüggemann, 1999, 2001; Hiley \& Yeadon, 2003a, 2003b; Yeadon \& Hiley, 2000). A gymnast performing giant circles rather behaves as a pendulum of smoothly varying length, much like the Botafumeiro, ${ }^{1}$ the famous giant censer (thurible) of the San-

\footnotetext{
${ }^{1}$ Oscillations (reaching an arc length of $65 \mathrm{~m}$ ) of this heavy-weight $(57 \mathrm{~kg}$ ) censer are produced by a group of men coordinating slack and pull on a cord wrapped around one of two coaxial rollers fixated onto the rotation axis located in the transept of the cathedral at a height of over $20 \mathrm{~m}$. The censer is suspended from a rope wrapped around the second roller, so that hauling in and letting out the cord leads to a shortening and lengthening of the pendulum length (see Sanmartin (1984), for details).
} 
tiago de Compostela Cathedral in North-Western Spain. As demonstrated by Stilling and Szyszkowski (2002) changes in pendulum length create a Coriolis inertia force that attenuates the motion when the length increases (i.e., when $\dot{r}>0$ ) and amplifies it when the length decreases (i.e., when $\dot{r}<0$ ). Equivalent conclusions with respect to the effects of length variation on rotational motion have been drawn by considering the principle of conservation of angular momentum (Walker, 1989). Including the torque produced by the Coriolis inertia force $(2 m r \dot{r} \dot{\theta})$ in Eq. (1) provides the equation of motion of a pendulum of varying length:

$$
\ddot{\theta}+2 \frac{\dot{r}}{r} \dot{\theta}+\frac{g}{r} \sin \theta=0 .
$$

It seems clear from this mechanical analysis that the gymnast has to control the relative rate of change of pendulum length $(\dot{r} / r)$ in order to succeed the giant circle. Of course, variation in pendulum length can only be brought about through changes in body configuration (i.e., by flexion and/or extension of the joints). Despite the fact that such changes are constrained by properties of the gymnast and the environment (Goldfield, Kay, \& Warren, 1993) - such as the architecture of the musculoskeletal system, the anthropometric properties of body segments, the material properties of the bar, the omnipresent gravitational field, and the task constraints contained in the code of points of the International Gymnastics Federation (2006) - a given pendulum length can be achieved through many different joint configurations. Yet, notwithstanding such redundancy in the available degrees of freedom, one of the hallmarks of perceptuo-motor expertise is the consistency of the movement patterns produced over repeated trials (Bardy \& Laurent, 1998; Franks, Weicker, \& Robertson, 1985; Hubbard \& Seng, 1954). While it has been proposed that such consistency in movement organization results from the repetitive execution of a predetermined plan of action (Schmidt, 1975; Tyldesley \& Whiting, 1975), it is perhaps better viewed as resulting from the repeated resolution of a similar problem of perception-action coupling (Bootsma \& van Wieringen, 1990; Peper, Bootsma, Mestre, \& Bakker, 1994; Zaal, Bootsma, \& van Wieringen, 1998). In this latter perspective movement behavior is taken to result from the operation of a law of control (Warren, 1988, 2006), dynamically linking relevant information sources to pertinent motor variables. Intercepting an object moving in the horizontal plane, for example, has been demonstrated to result from the regulation of locomotor velocity on the basis of changes in the object's bearing angle (Chardenon, Montagne, Laurent, \& Bootsma, 2004, 2005). If experts are characterized by their capacity to reliably implement such control laws, similar initial conditions should give rise to similar behavioral patterns.

In this light, examining the movement patterns produced under different task constraints allows identification of the invariant aspects of the perceptuo-motor solutions adopted. To this end, we examined how expert gymnasts spontaneously modified their movement patterns when performing backward giant circles on the high bar with additional weights attached at the level of the shoulders, waist, and ankles.

\section{Method}

\subsection{Participants}

Seven elite junior female gymnasts volunteered for participation in the experiment. Their ages ranged from 12 to 13 years (mean $12.4 \pm 0.5$ years), their standing height ranged from 1.35 to $1.54 \mathrm{~m}$ (mean $1.45 \pm 0.06 \mathrm{~m}$ ), and their body mass ranged from 28.0 to $47.5 \mathrm{~kg}$ (mean $38.1 \pm 6.9 \mathrm{~kg}$ ). Participants gave their informed consent prior to inclusion in the study, which was approved by the local ethics committee.

\subsection{Task}

Gymnasts performed backward giant circles on the high bar under different loading conditions. They were instructed to perform the backward giant circles with respect to the code of points of the International Gymnastics Federation (2006). According to this code, backward giant circles are 
to be executed with the legs together and without motion at the elbow or knee joints. In the framework of our experimental question, the gymnasts were instructed to conclude each swing in a vertical handstand position with minimal rotational velocity before initiating the next. After a few trials performed to allow for adaptation to the experimental situation, participants were requested to perform seven subsequent giant circles in each experimental condition. No temporal constraints were imposed during the session. Each gymnast could take as much rest as needed between conditions, each of which was spontaneously initiated, after a verbal signal from the experimenter. Data acquisition began at the signal.

\subsection{Apparatus}

Joint locations in two dimensions were recorded using a Basler video camera operating at a sampling frequency of $100 \mathrm{~Hz}$. The camera was placed perpendicularly to the plane of motion $8.50 \mathrm{~m}$ away from the right side of the participant at a height of $2.75 \mathrm{~m}$, such that the $x$-axis was aligned with the anteriorposterior direction, and the $y$-axis was aligned with the vertical direction. Six markers were attached to the participants' skin over the following right body side joints (Zatsiorsky, 2002): wrist (stylion), elbow (radiale), shoulder (acromion), hip (trochanterion), knee (tibiale), and ankle (lateral malleolus).

\subsection{Design}

Combining a control condition (unloaded) with three different load locations (with loads placed symmetrically on the upper arms near the shoulder joints, on the waist, or on the lower legs near the ankles) and two different magnitudes of load ( 2 or $4 \mathrm{~kg}$ total) gave rise to seven different experimental conditions. Participants performed all experimental conditions in a single experimental session lasting about $30 \mathrm{~min}$. The order of presentation of the conditions was randomized.

\subsection{Data analysis}

In order to examine stable performance, of the seven giant circles recorded only giant circles two to six were analyzed. Marker locations were digitized and transformed to 2D positions in the plane of motion using SIMI motion software. The position data were filtered using a fourth-order Butterworth low-pass filter with a zero phase shift and a $6 \mathrm{~Hz}$ net cut-off frequency (Winter, 1990). Masses and moment of inertia of the segments of individual participants were estimated using the anthropometric tables of Zatsiorsky and Seluyanov (1983).

Following Yeadon and Hiley (2000), body orientation was defined as the angle between the vertical and a line from the bar to the body center of mass (see Fig. 1). The body orientation angle $(\theta)$, shoulder angle $\left(\alpha_{1}\right)$, and hip angle $\left(\alpha_{2}\right)$ were estimated on the basis of displacement data using the convention illustrated in Fig. 1. Angular velocities were derived from angular position data. After segmentation of the five giant circles per condition based on body center of mass position with respect to the bar,

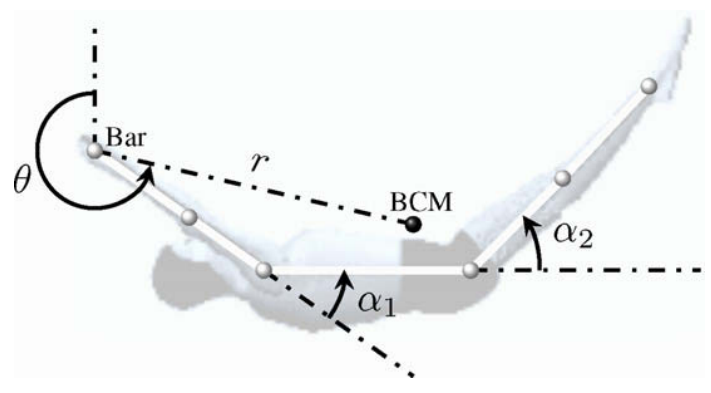

Fig. 1. Body segments model of a gymnast performing a backward giant circle. White dots represent the wrist, elbow, shoulder, hip, knee, and ankle markers and the black dot represents the location of the body center of mass (BCM). $\theta, \alpha_{1}$, and $\alpha_{2}$ are the three generalized coordinates. $r$ is the distance between the bar and the BCM (pendulum length). 
swing duration was calculated for each participant and each condition as the average time between two passes of the center of mass through the vertical above the bar.

\section{Results and discussion}

Notwithstanding the fact that 4-kg added load amounted to $10.5 \%$ of the total body weight on average (varying between $8.4 \%$ and $14.3 \%$ for individual participants), all seven gymnasts were able to perform the giant circles as requested in all loading conditions. During extended posture, the 2- and 4-kg loads gave rise to changes in the location of the body center of mass (BCM) relative to the axis of rotation of $-1.54 \pm 0.38$ and $-2.87 \pm 0.47 \mathrm{~cm}$, respectively, for loads placed at the shoulder, $+0.40 \pm 0.03$ and $+0.77 \pm 0.05 \mathrm{~cm}$, respectively, for loads placed at the waist, and $+5.74 \pm 0.60$ and $+10.69 \pm 0.88 \mathrm{~cm}$, respectively, for loads placed at the ankles. In line with the code of points of the International Gymnastics Federation (2006), gymnasts performed the giant circles with minimal motion at the elbow and knee joints; the observed range of motion was $4.7 \pm 1.2^{\circ}$ and $7.7 \pm 1.4^{\circ}$ for the elbow and knee joints, respectively.

For each individual participant, the pattern of movement revealed very little variability over repeated giant circles. Illustrating this observation, Fig. 2 presents the evolution over normalized time of BCM rotation angle $(\theta)$, shoulder angle $\left(\alpha_{1}\right)$, and hip angle $\left(\alpha_{2}\right)$ for five giant circles performed by a representative participant in the non-loaded control condition.

In this condition maximal hip extension (about $-33^{\circ}$ ) was reached at the end of the descent phase, at about $46 \%$ of swing duration ( $167^{\circ} \mathrm{BCM}$ rotation angle); maximal shoulder extension (about $-6^{\circ}$ ) was reached just after the lowest point, at about $50 \%$ of swing duration ( $190^{\circ} \mathrm{BCM}$ rotation angle): maximal hip flexion (about $45^{\circ}$ ) was reached during the upswing phase at about $62 \%$ of swing duration $\left(253^{\circ} \mathrm{BCM}\right.$ rotation angle), and maximal shoulder flexion (about $39^{\circ}$ ) was reached at about $70 \%$ of swing duration $\left(292^{\circ} \mathrm{BCM}\right.$ rotation angle).

\subsection{Swing duration}

A repeated measures analysis of variance (ANOVA) on swing duration with factors load location (ankle, waist, and shoulder level) and load magnitude ( 2 and $4 \mathrm{~kg}$ ) revealed a significant main effect
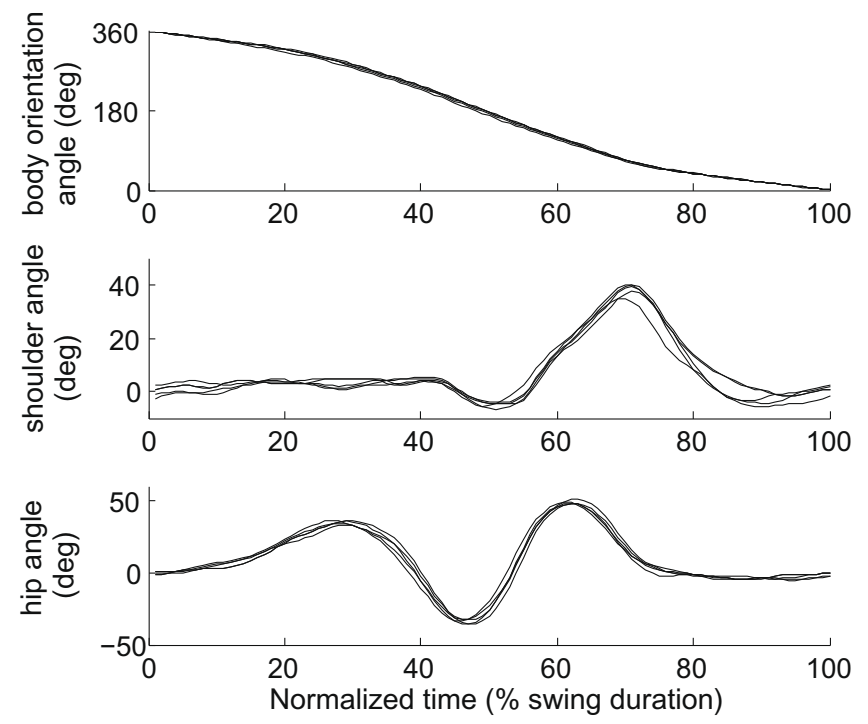

Fig. 2. Evolution over normalized time (percentage swing duration) of the body orientation angle $(\theta)$, shoulder angle $\left(\alpha_{1}\right)$, and hip angle $\left(\alpha_{2}\right)$ for the five giant circles of a representative participant in the non-loaded condition. 
of location, $F(2,12)=524.80, p<.001$, as well as a significant interaction between location and magnitude, $F(2,12)=18.29, p<.001$. As can be seen from Fig. 3, the magnitude and location of the loads systematically affected the duration of the swing. Post-hoc analysis (Tukey HSD) of the interaction revealed that swing duration varied $(p s<.001)$ over load locations and magnitudes, except when the loads were placed at the level of the waist.

The pattern of result obtained with respect to swing duration corroborated the interpretation of the gymnast circling on the high bar as a pendulum system. In the unloaded condition, swing duration was $1.92 \pm 0.16 \mathrm{~s}$. With the loads placed at the level of the ankles, thereby increasing the average distance between the rotation axis and the BCM (i.e., pendulum length) relative to the unloaded condition swing duration increased ( $1.99 \pm 0.14 \mathrm{~s}$ for the $2-\mathrm{kg}$ and $2.08 \pm 0.09 \mathrm{~s}$ for the $4-\mathrm{kg}$ loads). With the loads placed at the level of the shoulders, the average pendulum length and swing duration decreased $(1.78 \pm 0.17 \mathrm{~s}$ for the $2-\mathrm{kg}$ and $1.72 \pm 0.19 \mathrm{~s}$ for the $4-\mathrm{kg}$ loads). Placing the loads at waist level did not displace the location of the BCM to a significant degree and revealed swing durations close to that of the unloaded condition ( $1.90 \pm 0.08 \mathrm{~s}$ for the $2-\mathrm{kg}$ and $1.87 \pm 0.12 \mathrm{~s}$ for the 4 - $\mathrm{kg}$ loads).

\subsection{Peak joint extension and flexion}

While the pattern of shoulder and hip joint motion changed to a certain extent over loading conditions (see Fig. 4), it retained the qualitative characteristics described earlier.

\subsubsection{Magnitude of peak joint extension}

Repeated measures ANOVAs (two load magnitudes and three load locations) performed on the magnitude of joint angles at the moment of peak joint extension revealed a significant interaction between load location and magnitude, $F(2,12)=6.48, p<.05$, for the shoulder joint, but no statistically significant effects for the hip joint $(F s<1)$. In both cases the variations in the means over conditions were quite small (maximally $1.6^{\circ}$ for the shoulder and $1.2^{\circ}$ for the hip; see Fig. 4).

\subsubsection{Magnitude of peak joint flexion}

The ANOVAs performed on the magnitude of joint angles at the moment of peak joint flexion revealed significant main effects of load location $(F(2,12)=5.94, p<.05$, for the shoulder and $F(2$, $12)=49.45, p<.001$, for the hip) as well as significant interactions between load location and magnitude $(F(2,12)=10.97, p<.01$, for the shoulder and $F(2,12)=4.88, p<.05$ for the hip). As shown in

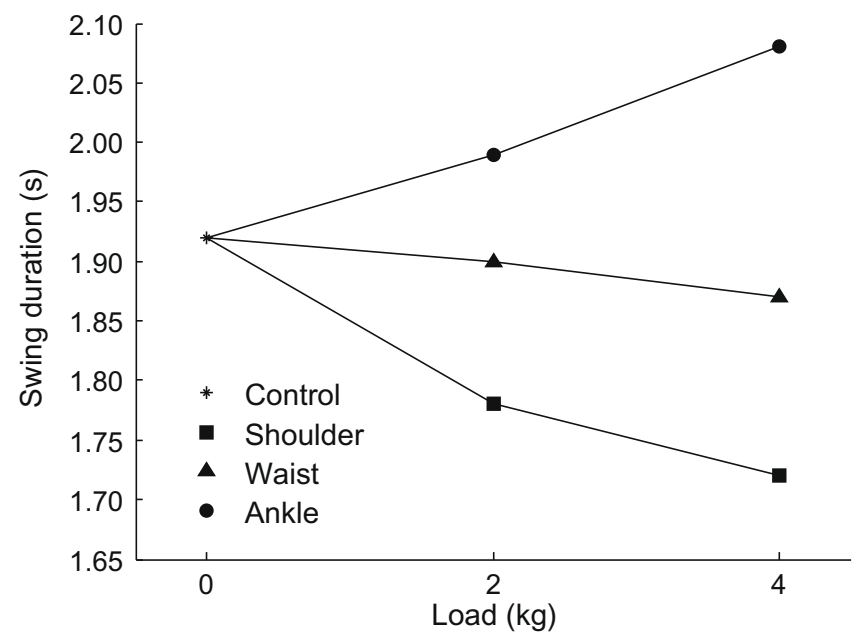

Fig. 3. Effects on total swing duration of adding 2- or 4-kg loads at the level of the shoulders (squares), waist (triangles), and ankles (circles). Swing duration in non-loaded $(0-\mathrm{kg})$ control condition is represented with an asterisk. 


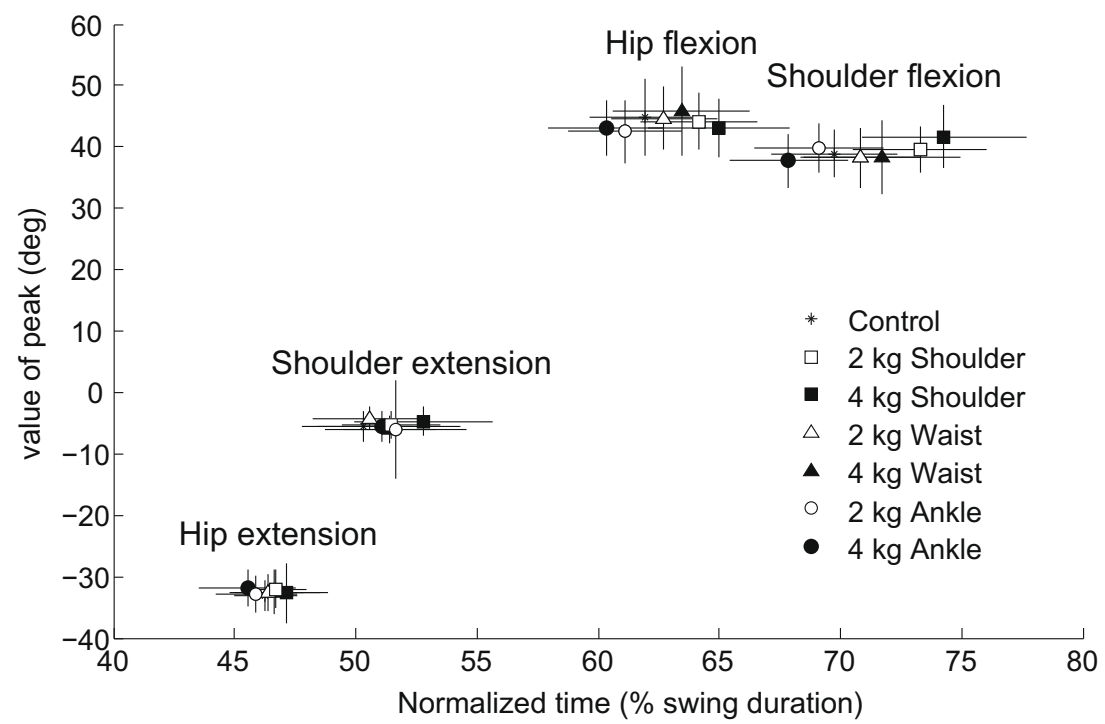

Fig. 4. Means and standard deviations (indicated by error bars) of the magnitudes of peak extension and flexion of the shoulder and hip joints as a function of their moment of occurrence expressed as the percentage of swing duration for the 2-kg (open symbols) and 4-kg (filled symbols) loads placed at the level of the shoulders (squares), waist (triangles), and ankles (circles). The non-loaded control condition is represented with asterisks.

Fig. 4 peak shoulder flexion was largest when the 4-kg load was placed at the level of the shoulders $\left(41.7^{\circ}\right.$, statistically different from all other conditions, $\left.p s<.01\right)$ and smallest when the $4-\mathrm{kg}$ load was placed at the level of the ankles $\left(37.8^{\circ}\right.$, statistically different from the 2-kg load at the ankles and both loads at the shoulder, $p s<.05$ ). Peak hip flexion was largest when the 4-kg load were placed at the waist level $\left(45.8^{\circ}\right.$, statistically different from all other conditions, $p s<.05$, except the $2-\mathrm{kg}$ load at the waist) and smallest when the loads were placed at the level of the ankles ( $42.5^{\circ}$ and $43.0^{\circ}$ for the 2- and 4-kg loads, respectively, statistically different from 2-kg load at the shoulder and the 2- and 4$\mathrm{kg}$ loads at the waist, $p \mathrm{~s}<.05$ ).

\subsubsection{Timing of peak joint extension}

ANOVAs performed on the percentage of swing time at which peak joint extension was reached revealed significant main effects of load location $(F(2,12)=9.48, p<.01$, for the shoulder and $F(2,12)=$ 5.07, $p<.05$, for the hip) as well as a significant interaction between load location and magnitude for the shoulder joint $(F(2,12)=5.72, p<.05)$. Peak shoulder extension was reached later $(52.8 \%)$ when the $4-\mathrm{kg}$ load was placed at shoulder level than for the other loading conditions ( $p s<.05$ ). Peak hip extension was reached earlier (45.5\%) when the $4-\mathrm{kg}$ load was placed at the level of the ankles ( $p \mathrm{~s}<.05)$ and later $(47.13 \%)$ when the $4-\mathrm{kg}$ load was placed at shoulder level $(p s<.05)$.

\subsubsection{Timing of peak joint flexion}

ANOVAs performed on the percentage of swing time at which peak joint flexion was reached revealed significant main effects of load location $(F(2,12)=77.11, p<.001$, for the shoulder and $F(2,12)=46.02, p<.001$, for the hip) as well as significant interactions between load location and magnitude for both the shoulder $(F(2,12)=6.07, p<.05)$ and hip $(F(2,12)=4.82, p<.05)$ joints. Peak shoulder flexion showed a clear pattern of change (see Fig. 4 ) over loading conditions ( $p s<.01$ ). With the loads placed at the level of the ankles, peak shoulder flexion was reached at $67.9 \%$ and $69.1 \%$ (for the 4- and 2-kg loads, respectively, ns). With the loads placed at waist level it was reached at $70.8 \%$ and $71.7 \%$ (for the 2- and 4-kg loads, respectively, $n s$ ) and with the loads placed at shoulder level it was reached at $73.3 \%$ and $74.3 \%$ (for the 2 - and $4-\mathrm{kg}$ loads, respectively, $n$ ). Peak hip flexion showed 
a similar pattern of change over loading conditions ( $p s<.01$ ), being reached at $61.1 \%$ and $60.3 \%$ (for the 2 - and 4-kg loads, respectively, ns) when the loads were placed at the level of the ankles, at $62.7 \%$ and $63.4 \%$ (for the 2 - and 4-kg loads, respectively, $n s$ ) when the loads were placed at waist level and $64.2 \%$ and $65.0 \%$ (for the 2- and 4-kg loads, respectively, $n s$ ) when the loads were placed at the shoulder level.

Taken together, as revealed by a global view of Fig. 4, these analyses demonstrated that load location and magnitude subtly but systematically influenced the timing and, to a lesser degree, the magnitude of peak joint extension and flexion. These effects were generally more pronounced for the shoulder joint than for the hip joint. Peak joint extension and peak joint flexion were reached earlier when the loading increased the distance between the rotation axis and the BCM and later when the loads decreased the distance between the rotation axis and the BCM. These changes in body configuration result in changes in the location of the $\mathrm{BCM}$ relative to the axis of rotation, that is, in pendulum length.

\subsection{Pendulum length}

In line with the results reported in the foregoing section with respect to peak joint extension and flexion, loading the gymnasts affected the variation of pendulum length $(r)$ in its timing rather than in its magnitude. The magnitude of pendulum length variation (calculated as the maximal minus minimal length observed during a swing) indeed revealed no statistically significant effects of load location or magnitude $(F s<1)$, for an average variation of $9.25 \pm 0.19 \mathrm{~cm}$. The moment of occurrence of the maximal pendulum length also did not vary over loading conditions $(F s<1.4)$, occurring at an average of $39.5 \pm 0.4 \%$ of swing duration $\left(118.8 \pm 2.1^{\circ} \mathrm{BCM}\right.$ rotation angle). However, the ANOVA on the moment of occurrence of minimal pendulum length revealed a significant main effect of load location, $F(2,12)=13.97, p<.001$, and a significant interaction between load location and magnitude, $F(2$, $12)=6.08, p<.05$. Minimal pendulum length was reached earliest (65.0\% of swing duration corresponding to $282.4^{\circ} \mathrm{BCM}$ rotation angle) when the $4-\mathrm{kg}$ load was placed at the level of the ankles and latest (71.7\% of swing duration corresponding to $294.0^{\circ}$ BCM rotation angle) when the 4-kg load was placed at the level of the shoulders ( $p s<.05)$.

\subsection{Regulation of pendulum length}

According to Eq. (2), what counts in terms of energy dissipating and restoring processes is not the length $(r)$ of the pendulum per se; it is the relative rate of change in pendulum length captured by $\dot{r} / r$ that matters. When $\dot{r}>0$ (i.e., the pendulum lengthens), energy is dissipated; when $\dot{r}<0$ (i.e., the pendulum shortens), energy is inserted. Fig. 5 presents the evolution of $r, \dot{r}$, and $\dot{r} / r$ as a function of the body rotation angle for the same five backward giant circles of the representative gymnast of Fig. 2 in the unloaded condition.

As can be seen from Fig. 5, the gymnast initially extends the body, with the equivalent pendulum reaching maximal length around a $\mathrm{BCM}$ rotation angle of $117^{\circ}$. The pendulum then shortens, reaching a sharp negative peak of $\dot{r} / r$ around $243^{\circ}$. Minimal pendulum length is reached at $282^{\circ}$, after which the gymnast extends before reaching the vertical handstand position.

Given that all gymnasts were able to perform the backward giant circles under all loading conditions, we must conclude that, under each condition, they succeeded in inserting the required amount of energy at the right place and time. The variations observed over different loading conditions in the magnitude and timing of shoulder and hip joint extension and flexion (Fig. 4) gave rise to variations in the pattern of change in pendulum length (Fig. 5). As a first step towards understanding the perceptuo-motor mechanism underlying the condition-specific regulations observed, we examined whether the sharp negative $\dot{r} / r$ peak, characteristic of the movement patterns of all gymnasts, occurred at a particular instance of the swing. To this end, we tested the invariance at the moment of the negative peak of $\dot{r} / r$ of the following candidate variables: (a) the percentage of swing duration, (b) the BCM rotation angle $\theta$, (c) the rate of change of BCM rotation angle $\dot{\theta}$, and (d) the ratio $\theta / \dot{\theta}$. The results of this analysis are presented in Table 1. 

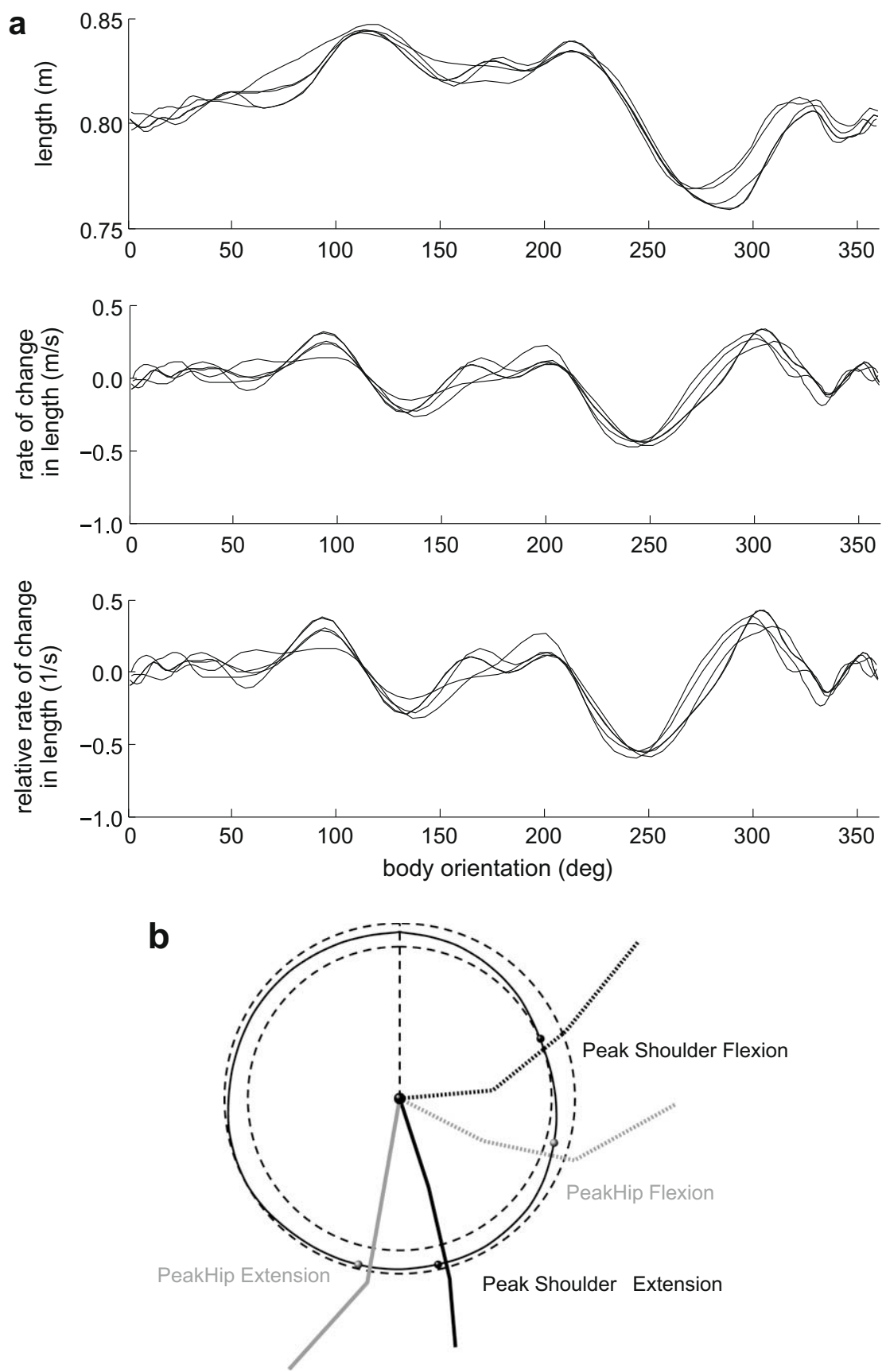

Fig. 5. (a) Evolution over body orientation angle $(\theta)$ of the length of the pendulum $(r)$, the rate of change in pendulum length $(\dot{r})$, and the relative rate of change in pendulum length $(\dot{r} / r)$ for the same five giant circles of the representative gymnast of Fig. 2 in the non-loaded condition. (b) Body configurations at the moment peak hip extension (grey continuous lines), peak shoulder extension (black continuous), peak hip flexion (grey dotted) and peak shoulder flexion (black dotted) and corresponding locations of the BCM (grey and black spheres), superimposed on a radial representation of equivalent pendulum length ( $r$, continuous black closed form). The dotted circles represent the shortest and longest pendulum length trajectories. 
Table 1

Effects of load location and magnitude on selected variables at the moment of occurrence of negative peak $\dot{r} / r$ : different lines refer, respectively, to time percentage of swing duration, BCM rotation angle $\theta$, BCM rotation speed $\dot{\theta}$, first-order time-to-closure $\theta / \dot{\theta}$. Note that for this latter measure, $\theta$ was defined as the gap remaining with the vertical (calculated as $360-\theta$ ). The different rows report for each loading condition the means and inter-individual standard deviations, together with ANOVA results.

\begin{tabular}{|c|c|c|c|c|c|c|c|c|c|c|}
\hline & \multirow[t]{2}{*}{ Unloaded } & \multicolumn{2}{|c|}{ Load at shoulder level } & \multicolumn{2}{|c|}{ Load at waist level } & \multicolumn{2}{|c|}{ Load at ankle level } & \multirow{2}{*}{$\begin{array}{l}\text { Location } \\
F(2,12)\end{array}$} & \multirow{2}{*}{$\begin{array}{l}\text { Magnitude } \\
F(1,6)\end{array}$} & \multirow{2}{*}{$\begin{array}{l}\text { Location } \times \text { magnitude } \\
F(2,12)\end{array}$} \\
\hline & & $2 \mathrm{~kg}$ & $4 \mathrm{~kg}$ & $2 \mathrm{~kg}$ & $4 \mathrm{~kg}$ & $2 \mathrm{~kg}$ & $4 \mathrm{~kg}$ & & & \\
\hline Time (\%) & $59.97 \pm 2.42$ & $62.17 \pm 2.12$ & $62.88 \pm 3.32$ & $60.97 \pm 3.09$ & $61.68 \pm 2.83$ & $58.85 \pm 2.60$ & $57.43 \pm 3.71$ & $42.14^{* * *}$ & 1.07 & $6.33^{*}$ \\
\hline$\theta(\mathrm{deg})$ & $243.0 \pm 5.4$ & $245.0 \pm 4.9$ & $247.3 \pm 7.3$ & $244.0 \pm 7.0$ & $245.9 \pm 6.5$ & $240.7 \pm 5.8$ & $238.8 \pm 5.8$ & $10.24^{* *}$ & 0.39 & $4.08^{*}$ \\
\hline$\dot{\theta}(\mathrm{deg} / \mathrm{s})$ & $262.8 \pm 3.3$ & $261.9 \pm 4.3$ & $261.7 \pm 2.4$ & $263.8 \pm 2.7$ & $263.1 \pm 3.3$ & $269.4 \pm 4.7$ & $274.1 \pm 3.9$ & $78.57^{* * *}$ & $12.48^{*}$ & $11.15^{* *}$ \\
\hline$\theta / \dot{\theta}(\mathrm{ms})$ & $445 \pm 28$ & $437 \pm 24$ & $431 \pm 35$ & $439 \pm 41$ & $433 \pm 31$ & $443 \pm 32$ & $441 \pm 28$ & 0.59 & 1.96 & 0.03 \\
\hline
\end{tabular}

${ }^{*} p<.05$.

*** $p<.01$

$p<.001$. 
As can be seen from this Table, at the moment of occurrence of negative peak $\dot{r} / r$ the percentage of swing duration, the body orientation and the rate of change of body orientation all revealed statistically significant variations as a function of loading conditions. Thus we may conclude that negative peak $\dot{r} / r$ does not occur (a) at an invariant time after launching the swing, (b) at an invariant orientation of the body, nor (c) at an invariant speed of rotation of the body. Interestingly the ratio $\theta / \dot{\theta}$ did not vary (to a statistically significant extent) over conditions. Although one has to be very careful in drawing conclusion from a non-significant effect, it is worth noting that the $\theta / \dot{\theta}$ ratio corresponds to the time remaining until the $\theta$ gap is closed if the current rate of closure were maintained (i.e., to $T C_{1}(\theta)$ in the terminology of Bootsma, Fayt, Zaal, and Laurent (1997)).

\section{General discussion}

In the present study we examined the movement patterns spontaneously produced by elite gymnasts performing backward giant circles on the high bar under different conditions of loading. In line with the recommendations of the code of points of the International Gymnastics Federation, the gymnasts kept the legs together and the elbow and knee joints in a fixed extended position, relying on motion at the level of the shoulder and hip joints to perform the action. Starting from a vertical handstand position above the bar, gymnasts produced a downward-swing movement pattern characterized by a peak hip extension reached shortly before and a peak shoulder extension reached shortly after the lowest point of the arc. The upward phase of the backward giant circle was characterized by a rapid flexion of both joints, with peak hip flexion occurring just before the body orientation reached the horizontal and peak shoulder flexion occurring slightly thereafter (see Figs. 2 and 5b). The high level of expertise of the participants was attested to by the consistency of the movement patterns produced over repeated trials (see Fig. 2). Together, several findings of the present study suggest that such consistency does not result from the repetitive execution of a predetermined action plan (Schmidt, 1975; Tyldesley \& Whiting, 1975). As shown in Fig. 4, the magnitude (2 or $4 \mathrm{~kg}$ ) and the location (shoulders, waist, or ankles) of the added loads subtly but systematically influenced the relative timing of movements produced. Moreover, the sharp negative $\dot{r} / r$ peak, characteristic of the behavior of all participants, did not occur at a particular (i.e., invariant) percentage of swing duration nor at a particular angle or rate of change of body orientation, varying systematically over loading conditions (see Table 1). Finally, the non-reported results of inverse dynamics procedures revealed large differences over loading conditions in the time-varying profiles of net forces and torques at shoulder and hip joints. Rather than interpreting the (consistency in the) movement patterns observed as resulting from a unique (learned) pattern of muscle activation, we suggest that it is better understood as resulting from the repeated resolution of a similar problem of perception-action coupling (Bootsma \& van Wieringen, 1990). Before addressing such coupling, let us first consider the mechanical constraints operating.

A physical analysis of the task suggested that a pendulum of variable length might constitute an appropriate mechanical analogy for the gymnast rotating around the bar. As a first corroboration of the pertinence of this analogy, swing duration was found to vary systematically with the magnitude and location of the loads added (see Fig. 3). Compared to the unloaded condition, placing a load at the level of the shoulders (thereby decreasing average pendulum length) led to a shorter swing duration while placing a load at the level of the ankles (thereby increasing average pendulum length) led to a longer swing duration.

As demonstrated by Stilling and Szyszkowski (2002), appropriate variations in pendulum length allow energy to be pumped into the system, thereby compensating for the energy lost to friction. With pendulum length defined as the distance between the axis of rotation (i.e., the bar) and the BCM of the gymnast, the observed changes in body configuration lead to changes in effective pendulum length. A second corroboration of the pertinence of the variable length pendulum analogy was provided by the finding that effective pendulum length varied as expected: it first slowly increased during the downward phase of the swing, then rapidly decreased during the first part of the upward phase of the swing, finishing with a slow increase in the last part (see Fig. 5). According to Eq. (2), however, it is not the length $(r)$ of the pendulum per se that matters. The amount of energy lost or gained is determined by the product of the relative rate of change of pendulum length $(\dot{r} / r)$ and the rate of change of 
body orientation $(\dot{\theta})$. Thus, during the slow lengthening phases only little energy is lost, while during the rapid shortening phase sufficient energy is pumped into the system so as to allow the gymnast to reach the final vertical handstand position.

Inspection of the variations in $\dot{r} / r$ as a function of body orientation angle for all gymnasts revealed the presence of a sharp negative peak occurring during the upward phase slightly before the horizontal was reached (see Fig. 5a lower panel for an example). Over the different conditions of execution, this peak did not occur at a constant moment in time, at a particular body orientation nor at a particular speed of rotation. Interestingly, however, the peak invariably occurred at a particular value of $\theta / \dot{\theta}$. The compound variable $\theta / \dot{\theta}$ corresponds to the time remaining until the gap $\theta$ will be closed if the current rate of closure $\dot{\theta}$ were to be maintained. In other words, $\theta / \dot{\theta}$ is the first-order time-to-closure $T C_{1}$ of the gap $\theta: T C_{1}(\theta)=\theta / \dot{\theta}$ (Bootsma et al., 1997). Of course, $T C_{1}(\theta)$ does not correspond to the "real" time-to-closure $(T C(\theta))$ of the gap because the rate of closure will in fact not remain constant. However, because the rate of closure will not only vary as a function of (foreseeable) environmental conditions (including gravity and friction forces) but also as a function of the future actions of the gymnast, "real" $T C(\theta)$ can only be determined a posteriori. As such, it does not constitute a temporal relation that can be used by the gymnast to control her ongoing action. $T C_{1}(\theta)$, on the other hand, exist throughout the action, unfolding over time and thereby constituting a robust and useful (first-order) temporal relation (Bootsma et al., 1997; Lee \& Young, 1985).

The fact that, over the different loading conditions, the sharp negative peak in $\dot{r} / r$ occurs around a fixed value of $T C_{1}(\theta)$ suggests that our gymnasts might have organized their behavior on the basis of $T C_{1}(\theta)$. Indeed, temporal relations of this kind have been identified as critical in the organization of a number of different actions (Bardy \& Laurent, 1998; Bootsma, Houbiers, van Wieringen, \& Whiting, 1991; Fayt, Bootsma, Marteniuk, MacKenzie, \& Laurent, 1997; Lee \& Reddish, 1981; Lee, Young, Reddish, Lough, \& Clayton, 1983; Peper et al., 1994; Warren, Young, \& Lee, 1986; Yilmaz \& Warren, 1995; Zaal et al., 1998). Further research will have to clarify (a) the perceptual (visual, proprioceptive, vestibular, ...) means by which the gymnasts may pick-up this first-order temporal relation and (b) the relation between the time specified and the organization of movement.

\section{References}

Arampatzis, A., \& Brüggemann, G. P. (1999). Mechanical energetic processes during the giant swing exercise before dismounts and flight elements on the high bar and the uneven parallel bars. Journal of Biomechanics, 32, 811-820.

Arampatzis, A., \& Brüggemann, G. P. (2001). Mechanical energetic processes during the giant swing before the Tkatchev exercise. Journal of Biomechanics, 34, 505-512.

Bardy, B. G., \& Laurent, M. (1998). How is body orientation controlled during somersaulting? Journal of Experimental Psychology: Human Perception and Performance, 24, 963-977.

Bauer, W. L. (1983). Swinging as a way of increasing the mechanical energy in gymnastic maneuvers. In H. Matsui \& K. Kobayashi (Eds.), Biomechanics VIII-B (pp. 1152-1159). Champaign, IL: Human Kinetics.

Bernstein, N. (1967). The coordination and regulation of movements. Oxford: Pergamon Press.

Bootsma, R. J., \& van Wieringen, P. C. W. (1990). Timing an attacking forehand drive in table tennis. Journal of Experimental Psychology: Human Perception and Performance, 16, 21-29.

Bootsma, R. J., Houbiers, M. H. J., van Wieringen, P. C. W., \& Whiting, H. T. A. (1991). Acquiring an attacking forehand drive: The effects of static and dynamic environmental information. Research Quarterly for Exercise and Sport, 62, 276-284.

Bootsma, R. J., Fayt, V., Zaal, F. T. J. M., \& Laurent, M. (1997). On the information-based regulation of movement: What Wann (1996) may want to consider. Journal of Experimental Psychology: Human Perception and Performance, 23, $1282-1289$.

Chardenon, A., Montagne, G., Laurent, G., \& Bootsma, R. J. (2004). The perceptual control of goal-directed locomotion: A common architecture for interception and navigation? Experimental Brain Research, 158, 100-108.

Chardenon, A., Montagne, G., Laurent, G., \& Bootsma, R. J. (2005). A robust solution for dealing with environmental changes in intercepting moving balls. Journal of Motor Behavior, 37, 52-64.

Fayt, V., Bootsma, R. J., Marteniuk, R. G., MacKenzie, C. L., \& Laurent, M. (1997). The effects of task constraints on the organization of interception movements. Journal of Sport Sciences, 15, 581-586.

Franks, I. M., Weicker, D., \& Robertson, D. G. E. (1985). The kinematics, movement phasing and timing of a skilled action in response to varying conditions of uncertainty. Human Movement Science, 4, 91-105.

Goldfield, E. C., Kay, B. A., \& Warren, W. H. (1993). Infant bouncing, the assembly and tuning of action systems. Child Development, 64, 1128-1142.

Hiley, M. J., \& Yeadon, M. R. (2003a). Optimum technique for generating angular momentum in accelerated backward giant circles prior to a dismount. Journal of Applied Biomechanics, 19, 119-130.

Hiley, M. J., \& Yeadon, M. R. (2003b). The margin for error when releasing the high bar for dismounts. Journal of Biomechanics, 36 , 313-319.

Hiley, M. J., \& Yeadon, M. R. (2005). Maximal dismounts from high bar. Journal of Biomechanics, 38, 2221-2227. 
Hubbard, A. W., \& Seng, C. N. (1954). Visual movements of batters. Research Quarterly, 25, 42-57.

International Gymnastics Federation (2006). Code of points for men's artistic gymnastics competitions. <http:// www.fedintgym.com/rules/>.

Lee, D. N., \& Reddish, D. E. (1981). Plummeting gannets: A paradigm of ecological optics. Nature, 293, 293-294.

Lee, D. N., \& Young, D. S. (1985). Visual timing in interceptive actions. In D. J. Ingle, M. Jeannerod, \& D. N. Lee (Eds.), Brain mechanisms and spatial vision (pp. 1-30). Dordrecht: Martinus Nijhoff.

Lee, D. N., Young, D. S., Reddish, D. E., Lough, S., \& Clayton, T. M. H. (1983). Visual timing in hitting an accelerating ball. Quarterly Journal of Experimental Psychology, 35A, 333-346.

Peper, C. E., Bootsma, R. J., Mestre, D. R., \& Bakker, F. C. (1994). Catching balls: How to get the hand to the right place at the right time. Journal of Experimental Psychology: Human Perception and Performance, 20, 591-612.

Sanmartin, J. R. (1984). O Botafumeiro: Parametric pumping in the middle ages. American Journal of Physics, 52, $937-944$.

Schmidt, R. A. (1975). A schema theory of discrete motor skill learning. Psychological Review, 82, 225-260.

Stilling, D. S. D., \& Szyszkowski, W. (2002). Controlling angular oscillations through mass reconfiguration: A variable length pendulum case. International Journal of Non-Linear Mechanics, 37, 89-99.

Tea, P. L., \& Falk, H. (1968). Pumping on a swing. American Journal of Physics, 36, 1165-1166.

Turvey, M. T. (1994). From Borelli (1680) and Bell (1826) to the dynamics of action and perception. Journal of Sport and Exercise Psychology, 16, S128-S157.

Tyldesley, D. A., \& Whiting, H. T. A. (1975). Operational timing. Journal of Human Movement Studies, 1, 172-177.

Walker, J. (1989). How to get a playground swing going: A first lesson in the mechanics of rotation. Scientific American, 260, 106-109.

Warren, W. H. (1988). Action modes and laws of control for the visual guidance of action. In O. G. Meijer \& K. Roth (Eds.), Complex movement behavior: 'The' motor-action controversy (pp. 339-380). Amsterdam: Elsevier.

Warren, W. H. (2006). The dynamics of perception and action. Psychological Review, 113, 358-389.

Warren, W. H., Young, D. S., \& Lee, D. N. (1986). Visual control of step length during running over irregular terrain. Journal of Experimental Psychology: Human Perception and Performance, 12, 259-266.

Winter, D. A. (1990). Biomechanics and motor control of human movement (2nd ed.). New York: John Wiley \& Sons.

Yeadon, M. R., \& Hiley, M. J. (2000). The mechanics of the backward giant circle on the high bar. Human Movement Science, 19, 153-173.

Yilmaz, E. H., \& Warren, W. H. (1995). Visual control of braking: A test of the tau hypothesis. Journal of Experimental Psychology: Human Perception and Performance, 21, 996-1014.

Zaal, F. T. J. M., Bootsma, R. J., \& van Wieringen, P. C. W. (1998). Coordination in prehension: Information-based coupling between reaching and grasping. Experimental Brain Research, 119, 427-435.

Zatsiorsky, V. (2002). Kinetics of human motion. Champaign, IL: Human kinetics.

Zatsiorsky, V., \& Seluyanov, V. (1983). The mass and inertia characteristics of main segments of the human body. In H. Matsui \& K. Kobayashi (Eds.), Biomechanics VIII-B (pp. 1152-1159). Champaign, IL: Human Kinetics. 